\title{
A HER2-mutant patient with late-stage duodenal adenocarcinoma benefited from anti-HER2 therapy and PD-1 inhibition: a case report
}

\author{
Zhiyu Wang, Wenwen Li, Yaning Wei, Lin An, Shenyong Su, Chenglin Xi, Kunjie Wang, Dan Hong, \\ Yan Shi \\ Department of Medical Oncology, Affiliated Hospital of Hebei University, Baoding, China \\ Correspondence to: Zhiyu Wang; Yan Shi. Department of Medical Oncology, Affiliated Hospital of Hebei University, Baoding 071000, China. \\ Email: 18931200826@189.cn; 11651735@qq.com.
}

\begin{abstract}
Duodenal adenocarcinoma (DA) is a subtype of small bowel adenocarcinoma (SBA). Compared with gastrointestinal cancers such as colorectal cancer and gastric cancer, SBA is less common. For patients with advanced and metastatic DA, chemotherapies are usually extrapolated from colorectal cancer and gastric cancer but the therapeutic effects remain undefined. Herein, we reported a 50-year-old female patient whom was diagnosed as stage IV DA with metastasis to both lungs and retroperitoneal lymph nodes. The next generation sequencing (NGS) using a panel consisting of 168 cancer related genes revealed amplification of the HER2/ERBB2 gene which has been a well-recognized therapeutic target among various tumor types. The anti-HER2 targeted therapy trastuzumab was used in combination with XELOX (oxaliplatin and capecitabine) as the first line treatment. The patient achieved partial response (PR) and had progression-free survival (PFS) of six months. After progressive disease (PD), the patient started the second line treatment with trastuzumab and PD1 inhibitors and remained stable disease (SD) with PFS for three months. The use of trastuzumab in neoadjuvant and adjuvant settings have been reported in sporadic cases. To the best of our knowledge, it is the first report to use anti-HER2 therapy and PD-1 inhibition as systemic therapy for advanced DA patients.
\end{abstract}

Keywords: Small bowel cancer; duodenal adenocarcinoma; trastuzumab; systemic therapy; case report

Submitted May 18, 2021. Accepted for publication Jul 22, 2021.

doi: 10.21037/jgo-21-311

View this article at: https://dx.doi.org/10.21037/jgo-21-311

\section{Introduction}

Small bowel adenocarcinoma (SBA) is a rare tumor type, accounting for less than $5 \%$ of gastrointestinal cancers (1). Duodenal adenocarcinoma (DA) is the most common subtype of SBA that is seen in more than half of SBA cases (1). Surgical resection of the primary tumor is the only curative treatment option for DA but it is not feasible for patients with advanced and metastatic disease (2). During clinical practice, chemotherapies for DA in the adjuvant or palliative treatment setting are usually extrapolated from colorectal cancer or gastric cancer (3). The HER2 protein overexpression and HER2 (also known as ERBB2) gene amplification have been observed in various cancer types (4).
Anti-HER2 therapies have been effectively applied to patients with breast cancer as well as colorectal cancer and gastric cancer (5), but its role in DA treatment was rarely reported. For patients with DA, anti-HER2 therapy named trastuzumab was used in neoadjuvant and adjuvant settings in sporadic cases (6). Herein, we reported a stage IV Chinese female HER2-mutant DA patient with lung metastasis benefited from trastuzumab in combination with chemotherapy. Trastuzumab was used in combination with XELOX as the first line treatment and was used with PD1 inhibitors as the second line treatment. To our knowledge, it is the first report to use trastuzumab and PD1 inhibitors as systemic therapy for metastatic DA. We present the 


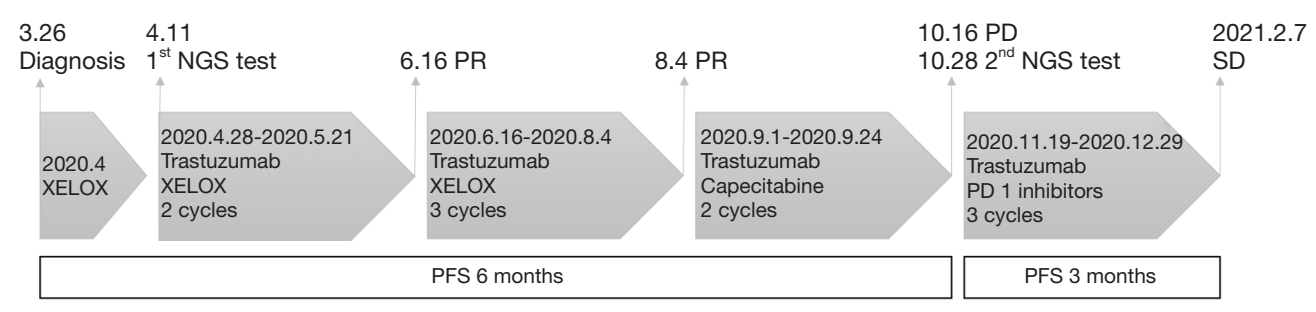

Figure 1 Treatment summary of the patient from diagnosis to last follow-up. PR, partial response; PD, progressive disease; NGS, next generation sequencing; SD, stable disease.

following article in accordance with the CARE reporting checklist (available at https://dx.doi.org/10.21037/jgo21-311).

\section{Case presentation}

A 50-year-old woman presented to the Affiliated Hospital of Hebei University due to cough with sputum and shortness of breath after physical activities. The patient had body mass index (BMI) of 20.7 and ECOG performance status of grade one. The patient reported no previous medical histories. During physical examination, no cardiopulmonary abnormalities were identified but mild tenderness was observed under the xiphoid process of upper abdomen without rebound tenderness or muscular tension. The positron emission tomography-computed tomography (PET-CT) indicated suspicious malignant lesions of the duodenum featured by horizontal section occupation and hypermetabolism. The hypermetabolism was also observed in retroperitoneal para-aortic lymph nodes and multiple pulmonary nodules in both lungs, indicating potential metastasis. Similar results were also obtained from enhanced CT scan on chest and abdomen. The magnetic resonance imaging (MRI) revealed irregular thickening of intestinal wall at the descending-horizontal part of the duodenal. High signals of diffusion-weighted imaging (DWI) were also observed. The duodenoscope identified circular occupation at the junction of descending-horizontal part of the duodenum. The patient was diagnosed as stage IV DA with metastasis to both lungs and retroperitoneal lymph nodes according to pathological evaluation on tissue samples obtained from duodenoscope and lung biopsy. The primary tumor at duodenum was adenocarcinoma with moderate differentiation. The immunohistochemistry indicated CK7(-), CK20(+), CDX2(+) and CA199(+). The metastatic tumor of the lung is also adenocarcinoma. Combined with clinical information and immunohistochemistry results [TTF-1(-), CK7(-), CK20(+), CDX2(+), Villin(+),
CA199(+)], the tumor was identified to be originated from digestive system.

The patient was treated with XELOX (oxaliplatin $200 \mathrm{mg}$ $\mathrm{d} 1$, capecitabine 1.5 po bid $\mathrm{d} 1-14$ ) as the first line therapy since April 2020 (Figure 1). Meanwhile, tumor samples and peripheral blood plasma of the patient were sent for next generation sequencing (NGS) using a panel consisting of 168 cancer related genes (Table 1). ERBB2 amplification was identified in tumors obtained from duodenum $(10 \%$ tumor content, $\mathrm{CN}: 3.7$ ), lung (40\% tumor content, $\mathrm{CN}: 9.0$ ) and in peripheral blood $(\mathrm{CN}: 2.3)$. Other mutations in ERBB2, TP53 and APC were identified in both tissue and blood samples. Both tissue and blood samples revealed microsatellite stable. Given the presence of ERBB2 amplification, the patient was subsequently administered trastuzumab in combination with XELOX for 5 cycles (Figure1). The treatment response evaluation performed on June and August 2020 indicated that the patient achieved PR (Figure 2). Gastrointestinal reactions (grade 1-2) and bone marrow suppression (grade 1-3) were observed during the treatment. Then, the patient received maintenance therapy with trastuzumab and capecitabine for 2 cycles. On October 2020, the patient had progressive disease (PD). The PFS of the patient under trastuzumab in combination with chemotherapy was six months.

The patient received the second NGS test which was performed in plasma sample and identified mutations in ERBB2, TP53 and APC. Since November 2020, trastuzumab in combination with PD1 inhibitors (camrelizumab for 2 cycles, tislelizumab for 1 cycle) were used as the second line treatment. No adverse effects were observed during the treatment. The recent treatment response evaluation performed on February 2021 indicated that the patient remained stable disease (SD) with PFS of 3 months (Figure 1). The patient currently achieved overall survival (OS) over 10 months and is still having regular followup. All procedures performed in studies involving human participants were in accordance with the ethical standards 
Table 1 Genetic profiling results of the patient

\begin{tabular}{lcccc}
\hline Sample & $\begin{array}{c}\text { 1st NGS test, duodenal } \\
\text { endoscope (tissue) }\end{array}$ & $\begin{array}{c}\text { 1st NGS test, lung } \\
\text { biopsy (tissue) }\end{array}$ & $\begin{array}{c}\text { 1st NGS test, peripheral } \\
\text { blood (plasma) }\end{array}$ & $\begin{array}{c}\text { 2nd NGS test, peripheral } \\
\text { blood (plasma) }\end{array}$ \\
\hline Tumor cell percentage & $10 \%$ & $40 \%$ & N/A & N/A \\
HER2 copy number (CN) & CN:3.7 & CN:9.0 & CN:2.3 & $5.45 \%$ \\
ERBB2 p.T862A & $45.46 \%$ & $85.92 \%$ & $11.17 \%$ & $0.52 \%$ \\
TP53 p.V143A & $11.73 \%$ & $71.53 \%$ & $1.70 \%$ & $0.58 \%$ \\
APC p.E771fs & $9.90 \%$ & $40.20 \%$ & $1.45 \%$ & $0.39 \%$ \\
APC p.E1554fs & $4.06 \%$ & $27.00 \%$ & $0.63 \%$ & MSS \\
MSI status & MSS & MSS & MSS \\
\hline
\end{tabular}

Allelic fractions are indicated in percentage. NGS, next generation sequencing; MSS, microsatellite stable; MSI, microsatellite instability.
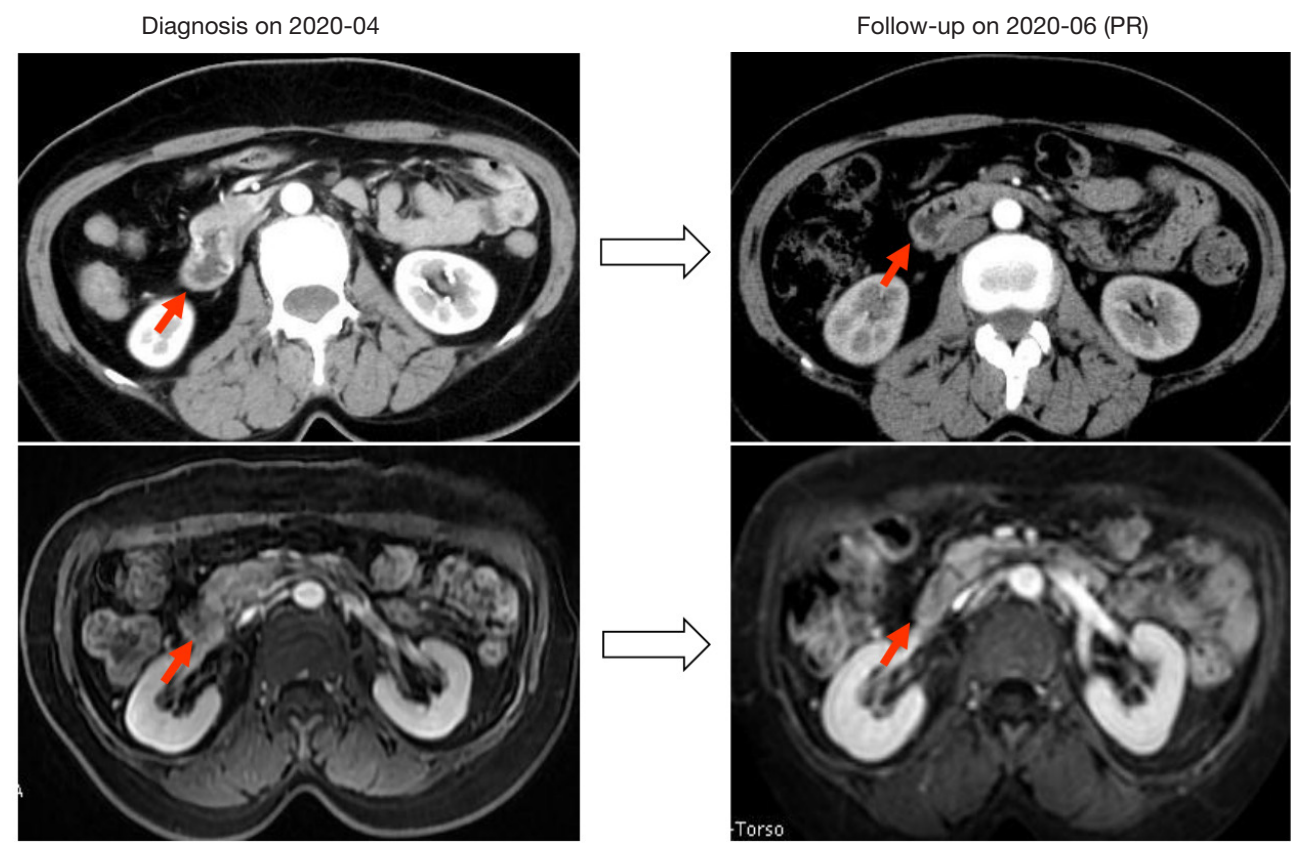

Figure 2 Treatment assessment by MRI when the patient archived PR. Tumors were indicated by the red arrow. MRI, magnetic resonance imaging; PR, partial response.

of the institutional and/or national research committee(s) and with the Helsinki Declaration (as revised in 2013). Written informed consent was obtained from the patient for publication of this case report and accompanying images. A copy of the written consent is available for review by the editorial office of this journal.

\section{Discussion}

Surgical resection is the only curative treatment option for
DA, while chemotherapies are usually used in the adjuvant or neoadjuvant settings (2). For patients with late-stage or metastatic DA, chemotherapies are used as palliative treatments for DA patients but the outcome remains poor. A meta-analysis based on 26 observational studies of DA reported that there were $71 \%$ patients received curative resection and 29\% patients received palliative treatment (2). Notably, the rate of 5 -year OS after curative resection was $46 \%$ but the 5 -year OS rate for palliative treatment was only $1 \%$ (2). Given the low prevalence of SBA and DA, there is 
a lack of research and convincing evidence to standardize and optimize chemotherapy regiments. Conventionally, chemotherapy regimens for DA were essentially the same as chemotherapy regiments for common gastrointestinal cancers such as colorectal cancer and gastric cancer. Although the tumor sites are close and risk factors are similar, their underlying molecular mechanisms could be different. A large-scale genomic study identified distinct genetic profiles of SBA comparing to colorectal cancer and gastric cancer (7). Recent published NCCN guidelines on SBA also indicated that SBA is a unique intestinal cancer at the molecular level (8).

We reported a stage IV DA case with lung metastasis. The NGS-based genetic profiling identified amplifications and mutations of HER 2 gene in both primary and metastatic sites. The HER2 gene encodes a transmembrane tyrosine kinase receptor. Amplification of HER2 results in protein overexpression which plays an important role during carcinogenesis. The anti-HER2 targeted therapy such as trastuzumab has been used for HER2-positive patients in several cancer types including breast cancer, colorectal cancer and gastric cancer. The role of HER 2 as a potential therapeutic target in SBA has been suggested by a number of genetic screening and profiling studies. A study based on a European database reported approximately $12 \%$ (10 of 83 ) of SBA cases carried genetic alterations of HER2 including mutations and amplifications (9). Another study also identified $8.3 \%$ (26 of 317) SBA cases with HER2 mutations (7). In our case, alterations of HER2 including amplification and point mutation p.T862A were presented in primary tumor site (duodenum), metastatic site (lung) and peripheral blood.

Previous study reported the use of anti-HER 2 targeted therapy in a HER2-positive patient with stage III DA. After surgical resection, the patient received 5-FU and trastuzumab for one year as adjuvant chemotherapy (10). In another HER2 amplified DA patient with liver metastasis, trastuzumab in combination with FOLFOX were used before surgery as neoadjuvant therapy and then the primary tumor was resected (11). In our case, trastuzumab was used in combination with XELOX as the first line treatment and was used with PD-1 inhibitors as the second line treatment. In HER-2 positive patients with breast cancer, PD1 inhibitor plus trastuzumab showed treatment benefit after trastuzumab resistance (12). To our knowledge, it is the first report to use trastuzumab in combination with XELOX or PD1 inhibitors as systemic therapy for a metastatic DA patient.
Although emerging studies reported the presence of HER2 amplification and mutation in SBA and DA patients, clinical use of anti-HER2 therapy is currently limited within a few sporadic cases with varied treatment intents and effects. Given the unique molecular signatures of DA compared with other gastrointestinal cancers, well-designed clinical studies are warranted to provide evidence-based indication for use of appropriate chemotherapy regimens.

\section{Acknowledgments}

The authors thank the patient and their families, medical and research staffs who participated in this study.

Funding: None.

\section{Footnote}

Reporting Checklist: The authors have completed the CARE reporting checklist. Available at https://dx.doi. org/10.21037/jgo-21-311

Conflicts of Interest: All authors have completed the ICMJE uniform disclosure form (available at https://dx.doi. org/10.21037/jgo-21-311). All authors declare that genomic profiling of the patient was performed at Burning Rock Biotech (Guangzhou, China). The authors have no other conflicts of interest to declare.

Ethical Statement: The authors are accountable for all aspects of the work in ensuring that questions related to the accuracy or integrity of any part of the work are appropriately investigated and resolved. All procedures performed in studies involving human participants were in accordance with the ethical standards of the institutional and/or national research committee(s) and with the Helsinki Declaration (as revised in 2013). Written informed consent was obtained from the patient for publication of this case report and accompanying images. A copy of the written consent is available for review by the editorial office of this journal.

Open Access Statement: This is an Open Access article distributed in accordance with the Creative Commons Attribution-NonCommercial-NoDerivs 4.0 International License (CC BY-NC-ND 4.0), which permits the noncommercial replication and distribution of the article with the strict proviso that no changes or edits are made and the original work is properly cited (including links to both the 
formal publication through the relevant DOI and the license). See: https://creativecommons.org/licenses/by-nc-nd/4.0/.

\section{References}

1. Aparicio T, Zaanan A, Mary F, et al. Small Bowel Adenocarcinoma. Gastroenterol Clin North Am 2016;45:447-57.

2. Meijer LL, Alberga AJ, de Bakker JK, et al. Outcomes and Treatment Options for Duodenal Adenocarcinoma: A Systematic Review and Meta-Analysis. Ann Surg Oncol 2018;25:2681-92.

3. Zouhairi ME, Venner A, Charabaty A, et al J. Small bowel adenocarcinoma. Curr Treat Options Oncol 2008;9:388-99.

4. Ménard S, Pupa SM, Campiglio M, et al. Biologic and therapeutic role of HER2 in cancer. Oncogene 2003;22:6570-8.

5. Hudis CA. Trastuzumab--mechanism of action and use in clinical practice. N Engl J Med 2007;357:39-51.

6. Sacdalan DB, Mendoza MJ, Vergara JP, et al. Beyond bevacizumab: a review of targeted agents in metastatic small bowel adenocarcinoma. Med Oncol 2020;37:106.

7. Schrock AB, Devoe CE, McWilliams R, et al. Genomic Profiling of Small-Bowel Adenocarcinoma. JAMA Oncol

Cite this article as: Wang Z, Li W, Wei Y, An L, Su S, Xi C, Wang K, Hong D, Shi Y. A HER2-mutant patient with latestage duodenal adenocarcinoma benefited from anti-HER2 therapy and PD-1 inhibition: a case report. J Gastrointest Oncol 2021;12(4):1939-1943. doi: 10.21037/jgo-21-311
2017;3:1546-53.

8. Benson AB, Venook AP, Al-Hawary MM, et al. Small Bowel Adenocarcinoma, Version 1.2020, NCCN Clinical Practice Guidelines in Oncology. J Natl Compr Canc Netw 2019;17:1109-33.

9. Laforest A, Aparicio T, Zaanan A, et al. ERBB2 gene as a potential therapeutic target in small bowel adenocarcinoma. Eur J Cancer 2014;50:1740-6.

10. Braga VM, de Oliveira MB, Netto CC, et al. Human Epidermal Growth Factor Receptor 2-Positive Duodenal Adenocarcinoma: A Case Report and Review of the Literature. Case Rep Oncol 2015;8:285-9.

11. Hamad A, Singhi AD, Bahary N, et al. Neoadjuvant Treatment With Trastuzumab and FOLFOX Induces a Complete Pathologic Response in a Metastatic ERBB2 (HER2)-Amplified Duodenal Cancer. J Natl Compr Canc Netw 2017;15:983-8.

12. Loi S, Giobbie-Hurder A, Gombos A, et al. Pembrolizumab plus trastuzumab in trastuzumab-resistant, advanced, HER2-positive breast cancer (PANACEA): a single-arm, multicentre, phase 1b-2 trial. Lancet Oncol 2019;20:371-82.

(English Language Editor: B. Draper) 